\title{
Accuracy issues of the existing thermospheric wind models: can we rely on them in seeking solutions to wind-driven problems?
}

\author{
M. F. Larsen ${ }^{1}$ and C. G. Fesen ${ }^{1, *}$ \\ ${ }^{1}$ Department of Physics \& Astronomy, Clemson University, Clemson, SC, USA \\ * currently at: National Science Foundation, Arlington, VA, USA
}

Received: 4 December 2008 - Revised: 28 April 2009 - Accepted: 29 April 2009 - Published: 8 June 2009

\begin{abstract}
We address the question of the ability of empirical and general circulation model neutral wind profiles in the lower thermosphere to reproduce the observed characteristics of the winds in that part of the atmosphere. The winds in that altitude range are critical for electrodynamic processes, but evaluations of the model winds are generally difficult because of the sparse observational data, which makes an evaluation of the wind predictions over large areas difficult or impossible. In this paper, we use a recently identified characteristic of the winds in the lower thermosphere, namely the enhanced winds and strong shears between 95 and $115 \mathrm{~km}$ altitude, as a test of the models, at least in a statistical sense. Our results show that the Horizontal Wind Model (HWM) significantly underestimates the maximum winds and shears in the lower thermosphere, although it has reasonable agreement with the average winds. The NCAR general circulation model used in this study also underestimates the maximum winds and shears significantly when run with standard resolution, as well as producing an unrealistic increase of the wind speed with height. The agreement between the model and the observations improves significantly however, in a statistical sense, when the altitude resolution is increased. The improved height resolution in the model appears to produce a greater improvement in the model predictions than any of the other factors that we examined, such as improving the geomagnetic forcing or the forcing at the lower boundary.
\end{abstract}

Keywords. Ionosphere (Ionosphere-atmosphere interactions) - Meteorology and atmospheric dynamics (Thermospheric dynamics; Waves and tides)

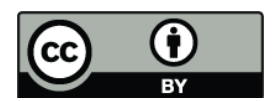

Correspondence to: M. F. Larsen (mlarsen@clemson.edu)

\section{Introduction}

The neutral winds are an important driver or a critical parameter in nearly all electrodynamic and plasma physics processes in the mid- and low-latitude ionosphere. The background wind profiles, for example, are a critical factor in determining which gravity wave modes can reach the ionosphere and where their effects are manifested (see, e.g., Vadas, 2007). The winds and shears are also critical factors in the development of sporadic $\mathrm{E}$ and potentially in the evolution of the structure within the plasma instability structures associated with that phenomenon (see, e.g., Larsen et al., 2007, and discussion therein). The winds and shears furthermore drive important current systems such as the Sq dynamo and the equatorial electrojet. There are only a few techniques available for measuring the neutral winds in the thermosphere and few locations and/or times when such measurements are made, either routinely or as part of special observing periods. The community therefore relies heavily on either empirical or first-principle models to provide estimates of the winds that can be used in the analysis of electrodynamic or plasma processes. The sparseness of the available wind measurements that makes it necessary to rely on the models also makes it difficult to make detailed assessments of the quality of the model winds, but a qualitative assessment based on the ability of the models to reproduce easily recognizable features of the observational data is possible. In this paper we present an analysis of the model winds in the E-region by comparing the distribution of winds and shears from the models with those from a large data set of sounding rocket chemical release wind profiles. Specifically, the enhanced circulation in the lower thermosphere that has been identified in the database of sounding rocket neutral wind measurements (Larsen, 2002) provides a convenient marker that can be used as a basis for comparison with the models. Our focus in this study is therefore on the winds in the lower E-region.

Published by Copernicus Publications on behalf of the European Geosciences Union. 


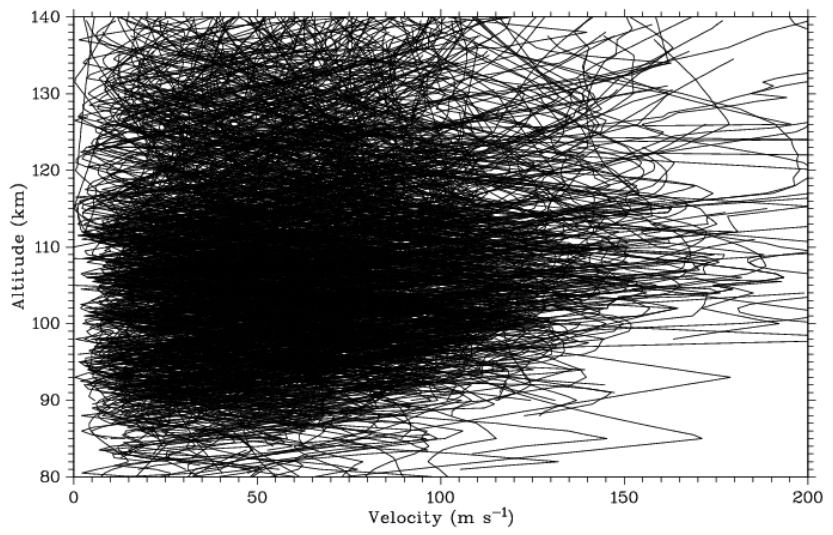

Fig. 1. Superposition of more than 500 mid- and low-latitude neutral wind magnitude profiles measured with the chemical release technique from sounding rockets since the late 1950s.

Assessments of thermospheric wind models have been difficult because detailed wind profile measurements are generally only available at isolated locations in limited height ranges and over limited periods. It is difficult therefore to determine if discrepancies between the model predictions and the observed winds are localized, transient effects or if they represent a fundamental shortcoming of the model. The approach that has often been taken therefore is to fit global wave modes, usually tides, to the observations and compare those results with theory. Many of the problems of interest for ionospheric physics, however, deal with localized phenomena for which models based on fits of global-scale modes may be inadequate for a full understanding of the relevant physical processes.

In the next section we describe the rocket wind measurement characteristics and follow that with a comparison of the rocket and empirical model results. We conclude with a comparison of the winds from the first-principles National Center for Atmospheric Research (NCAR) general circulation model (GCM) with the overall rocket wind data set and with observations made on two individual nights.

\section{Chemical release wind data set}

Since 1958, more than 500 measurements of the neutral wind profiles have been made with the chemical release technique from sounding rockets. The measurement is based on the release of a chemical tracer trail that is visible from the ground over a period of several minutes. Optical tracking of the trails provides wind profiles with good height resolution that is generally $0.5 \mathrm{~km}$ or better and relatively small uncertainties, generally $5 \mathrm{~m} \mathrm{~s}^{-1}$ or better, in the wind speed estimates. Characteristics of the data set were described in detail by Larsen (2002). Since the publication of that report, new measurements and the addition of older measurements that were not included in the original data set have increased the total number of profiles by approximately $20 \%$.

The conclusion of the analysis by Larsen (2002) was that there is a region of enhanced winds in the altitude range between 95 and $115 \mathrm{~km}$, as shown in Fig. 1, which represents a superposition of all the chemical release wind magnitude profiles. The large winds and associated large shears above and below the wind maximum are common features of the wind profiles in that altitude range and at all mid- and lowlatitude locations. The feature was initially identified in the rocket data, but has been verified in other data sets as well, notably the Doppler sodium lidar data obtained by the University of Illinois in New Mexico and Hawaii. The details of the comparison between the lidar winds and the chemical release data were described in the Masters thesis by Zhou (2005). The main result of the comparison is shown in Fig. 2, which represents a superposition of zonal and meridional lidar wind profiles over a seven-year period from 1998 to 2004, plotted in red, on top of the chemical release wind component profiles. The lidar data are generally only reliable up to an altitude of $\sim 105 \mathrm{~km}$, but the agreement below that height is good, and both data sets show the increase in the winds with height and similar maximum wind speeds. Larsen et al. (2003) also made a detailed comparison of a simultaneous rocket and lidar wind measurement made in New Mexico.

The enhanced winds and shears in the lower thermosphere evident in the rocket and lidar data would be important in wind-driven problems related to mid- and low-latitude electrodynamics, but the question is whether the available models are capable of reproducing such features, either on individual nights or in a statistical sense.

\section{Empirical model comparison}

The Horizontal Wind Model (HWM) (Hedin et al., 1991) has been used extensively for the analysis of thermospheric dynamics and ionospheric electrodynamics, and we will use it here for the comparison with the rocket winds. Specifically, for each wind profile in the rocket data set in Fig. 1, a corresponding wind profile for the same time, location, and altitude range was calculated with HWM. The results are shown in Fig. 3. The model clearly has smaller wind speeds and shears than the observational data set and, in particular, the increase in wind speed with height occurs much higher in the empirical model than in the observations.

A comparison of the zonal and meridional wind components was also carried out, and the results are shown in Fig. 4. The lower panels show the average wind profiles for the rocket winds, as well as the standard deviation profiles for each component. The upper panels show the superposition of all the HWM zonal and meridional wind profiles. The agreement between the meridional profiles is quite good. Both the model and observational data set show a trend from 


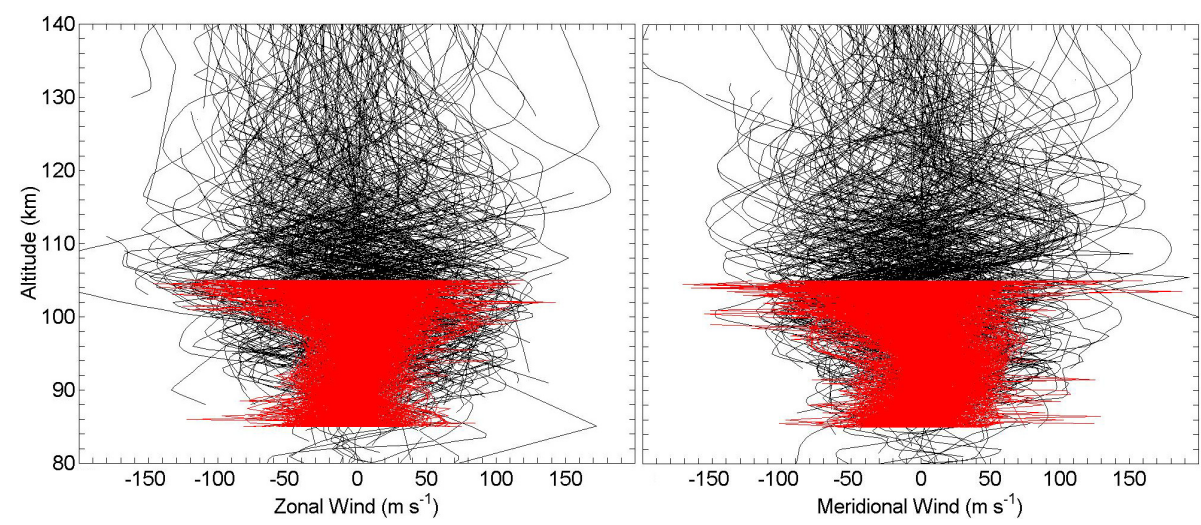

Fig. 2. Superposition of zonal and meridional wind profiles obtained with the Doppler sodium lidar over a seven-year period (red curves) and the wind components obtained from the chemical release wind measurements (black curves).

northward winds at lower altitudes to southward winds at the upper altitudes. The small enhancement in the southward winds between 100 and $105 \mathrm{~km}$ is also reproduced in the model winds. For the zonal wind, the observed and model winds both show a trend from more eastward winds at lower altitudes to more westward winds at higher altitudes. The HWM winds have a much larger spread in the zonal component than in the meridional component above $\sim 110 \mathrm{~km}$ while in the observations the distribution is similar for both components. The HWM winds therefore have the same general variation with height as the mean rocket winds but have an anisotropy in the wind components that is not present in the observations.

The techniques available for neutral wind measurements in the lower thermosphere are very limited. Although a variety of data sources is included in the formulation of the empirical model, the incoherent scatter radar technique is notable in that it provides the only wind profile measurements over an extended altitude range with good height resolution and good daytime temporal coverage. The technique is not a direct neutral wind measurement but requires a solution of the ion momentum equation with various assumptions. The incoherent scatter radar measurements of the neutral winds are a primary source of neutral wind information in the lower E-region.

The most extensive set of neutral wind observations with the incoherent scatter technique is from the Lower Thermosphere Coupling Study (LTCS) (Fesen et al., 1993) which included a large number of observational campaigns with extended ISR wind measurements that were designed to provide a better global specification of the tidal modes, among other goals. The measurements covered a range of seasons and different geomagnetic activity levels. We have plotted the superposition of the various Arecibo Observatory ISR wind profile measurements obtained during the LTCS campaigns in Fig. 5. The zonal wind components in the left panel and the meridional wind components in the right panel can be

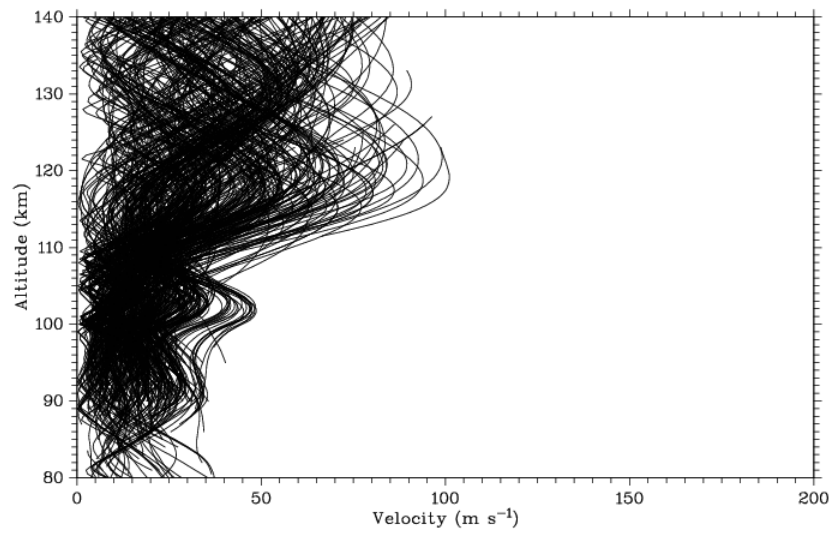

Fig. 3. The Horizontal Wind Model neutral wind magnitude profiles corresponding to the profiles shown in Fig. 1.

compared directly to the rocket and lidar wind components plotted in Fig. 2. In general, the maximum wind speeds in the two data sets are comparable. There is an indication in the ISR data of the enhanced circulation feature that maximizes near $105 \mathrm{~km}$, but the winds increase rapidly both above and below so that there is little evidence of the general vertical trend in the winds that is evident in both the rocket and lidar winds.

\section{First-principles model comparison}

There are several first-principles models that have been used extensively in modeling the general circulation of the thermosphere. The best comparison between the observations and a general circulation model would be a study in which model runs are carried out for conditions that correspond specifically to the times and locations of each profile plotted in Fig. 1, but that requires significant computational 

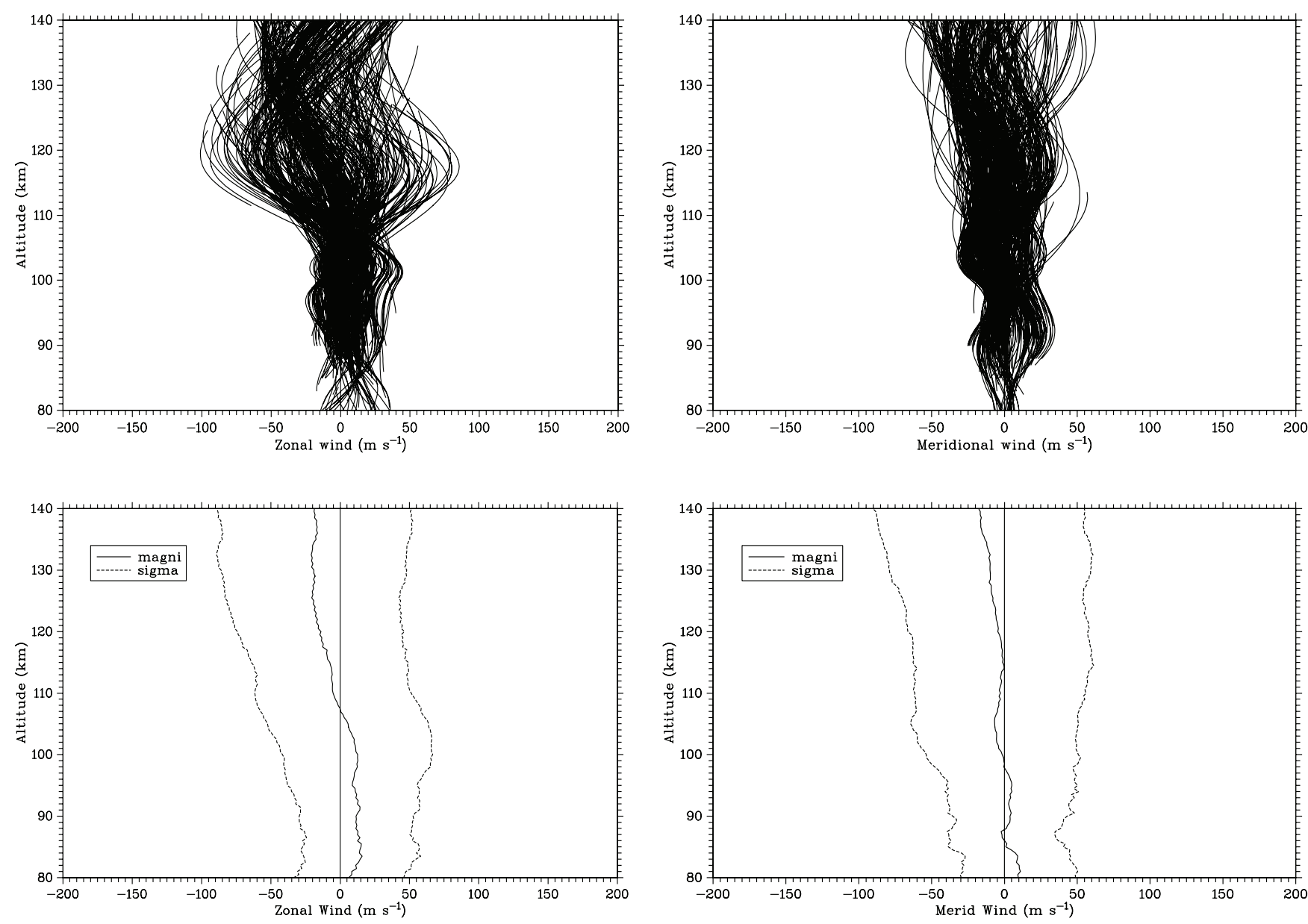

Fig. 4. Zonal (left panel) and meridional (right panel) HWM wind components for the profiles shown in Fig. 3 are shown in the top panels. The curves in the lower panels show the mean and standard deviations for the chemical release wind components.

resources. The study by Larsen (2002) indicates, however, that the enhanced winds in the 95 to $115-\mathrm{km}$ altitude range occur in all seasons and at all locations. Even the winds from one location, such as the lidar site in New Mexico, shows a distribution of wind speeds that is similar to that found in the rocket data set, suggesting that a meangingful study can still be carried out by comparing the distribution of winds from a more limited model run with the observations. Here we focus specifically on runs that were made with the National Center for Atmospheric Research (NCAR) ThermosphereIonosphere-Mesosphere-Electrodynamics General Circulation Model (TIME-GCM) (Roble and Ridley, 1994) for the year 1993. The model includes a self-consistent treatment of the ionosphere and electrodynamics. The lower boundary is at $30 \mathrm{~km}$ and the upper boundary is near $500 \mathrm{~km}$. The model is hydrostatic and is formulated in isobaric coordinates. The resolution is two grid points per scale height for a total of 45 pressure levels and a horizontal resolution of $5^{\circ}$ latitude by $5^{\circ}$ longitude. Four separate one-year model runs were car- ried out for conditions appropriate to 1993. The first used a fixed solar and auroral forcing, i.e., without daily variations in the magnetic activity. The second run used the daily indices of solar and geomagnetic forcing for 1993. The third run was similar to the second but also included the NCEP tropospheric weather analysis to force the lower boundary. The fourth run used the daily indices for March 1993, the NCEP forcing, and a doubled height resolution, i.e., 90 pressure levels (4 grid points per scale height) instead of the standard 45 levels.

The superposition of wind profiles for all grid points between $52.5^{\circ} \mathrm{S}$ and $52.5^{\circ} \mathrm{N}$, i.e., all mid- and low-latitude grid points, on 21 March is shown in Fig. 6 for each of the four model runs. Specifically, the model runs were saved once per hour. The model runs were for the entire year, but the profiles included in the plot are for a single day, namely 21 March. The data set includes profiles with $5^{\circ}$ longitude spacing ( 73 profiles), $5^{\circ}$ latitude spacing between $52.5^{\circ} \mathrm{S}$ and $52.5^{\circ} \mathrm{N}$ (22 profiles), and 25 hourly values for a total of 
40150 profiles. Only the zonal wind results are shown here but are qualitatively similar to the results for the meridional component. The results for the first run with constant solar and geomagnetic forcing are shown in the upper lefthand panel. The resulting wind profiles have smaller wind speeds below $\sim 115 \mathrm{~km}$ with a significant increase in the wind speeds above that altitude. There is also a narrow enhancement in the wind speeds that peaks near $95 \mathrm{~km}$. The agreement between the model winds and the chemical release data set is generally poor in this case. Specifically, the rapid increase of the winds with altitude above $115 \mathrm{~km}$ is significantly different from the observed winds, and the lower altitude peak in the $90-100 \mathrm{~km}$ altitude range shows an asymmetry that is not present in the observations and also occurs at too low an altitude.

The results for the second run with realistic day-to-day variability in the solar and geomagnetic forcing are shown in the upper righthand panel. The maximum wind speeds below $115-\mathrm{km}$ altitude are reduced overall, the wind enhancement near $95 \mathrm{~km}$ has been eliminated, and the rapid increase in the wind speeds with height above $115 \mathrm{~km}$ is still present, although reduced in magnitude.

The zonal wind profiles for the third run with variable solar and geomagnetic forcing and NCEP forcing at the lower boundary are shown in the lower lefthand panel. The rapid increase in the winds with height above $115 \mathrm{~km}$ has been eliminated in this run and the maximum wind speeds are comparable to observed maximum winds in that altitude range. The results are therefore in better agreement with the observations in this case. These wind profiles have an enhanced wind maximum between 100 and $110 \mathrm{~km}$, although there is a strong asymmetry between the eastward and westward component that is not evident in the observations.

In the fourth run, shown in the lower righthand panel, the variable solar and geomagnetic forcing were included, as well as the NCEP forcing of the lower boundary, but in addition, the height resolution was doubled, with four instead of two grid points per scale height. The difference between this run and the other three runs is significant. The maximum wind speeds have increased significantly and have magnitudes that are in good qualitative agreement with the observed wind magnitudes. In addition, the height variation of the winds is also in good agreement with the observed variations, with winds peaking in the lower thermosphere and realistic decreases in the winds above and below the altitude of the peak. The enhanced winds are symmetric, again in agreement with the observed wind characteristics. The most significant discrepancy between the model and observations in this case is that the enhanced winds in the model peak near $100 \mathrm{~km}$, i.e., approximately $5 \mathrm{~km}$ below the peak winds in the chemical release data set. In general, the doubled height resolution significantly improved the agreement with the observations and suggests that the model can produce winds with realistic wind speeds and shears in the mesosphere-lowerthermosphere (MLT) and lower E-region.
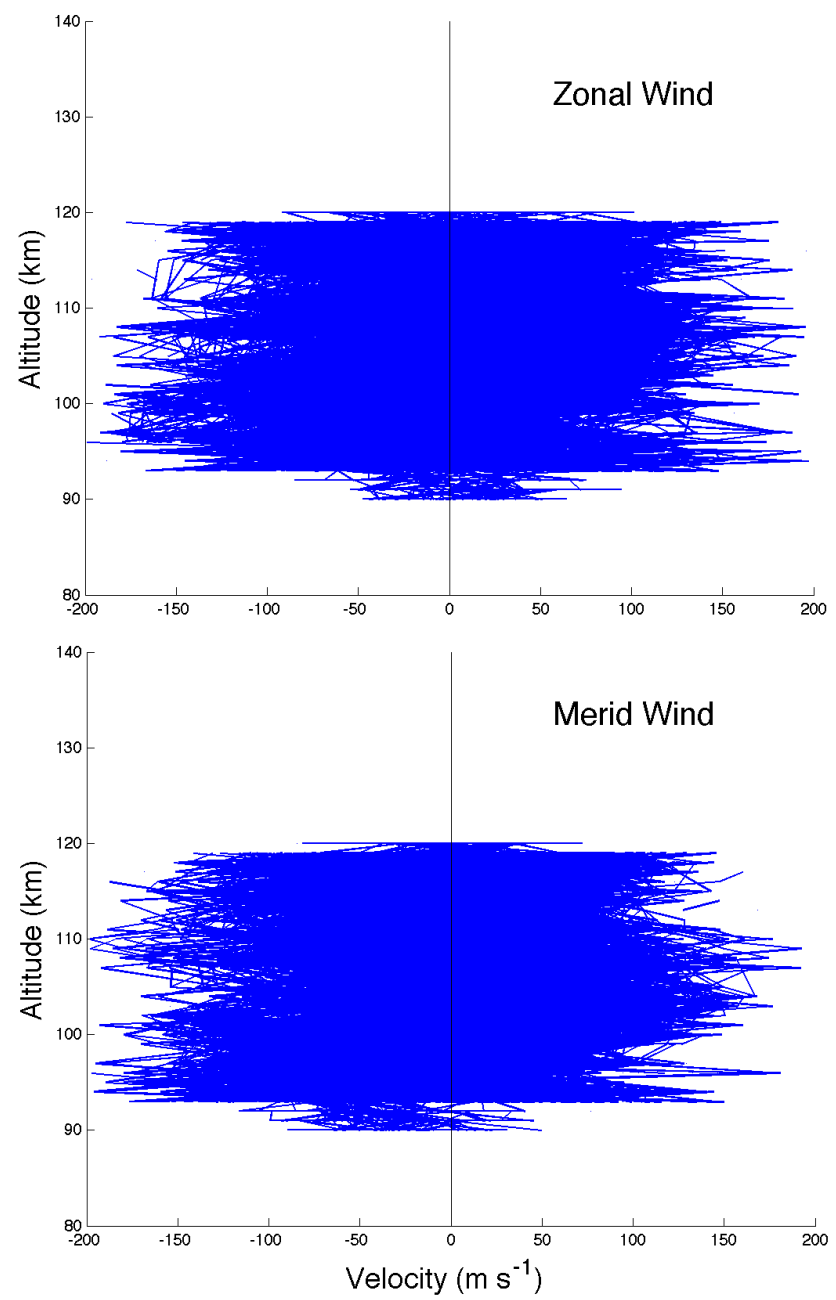

Fig. 5. Superposition of the zonal and meridional wind profiles obtained with the Arecibo Observatory incoherent scatter radar during the Lower Thermosphere Coupling Study observation periods.

The details of the observed and model wind distributions is shown in Fig. 7 with the histogram of maximum wind speeds shown in the upper panel and the histogram of maximumwind layer heights in the lower panel. The dashed curves show the distributions for the rocket observations, and the solid curves show the distributions for the doubled height resolution model run. The peak in the model wind speeds occurs in the interval between 60 and $80 \mathrm{~m} \mathrm{~s}^{-1}$, while the peak in the observed wind speeds occurs in the interval between 100 and $120 \mathrm{~m} \mathrm{~s}^{-1}$. The layer height with maximum winds in the observations are found most frequently in the 100 to $105 \mathrm{~km}$ altitude range. The model run has a broader height distribution for the maximum-wind layer heights than the observations and is shifted toward lower heights. 


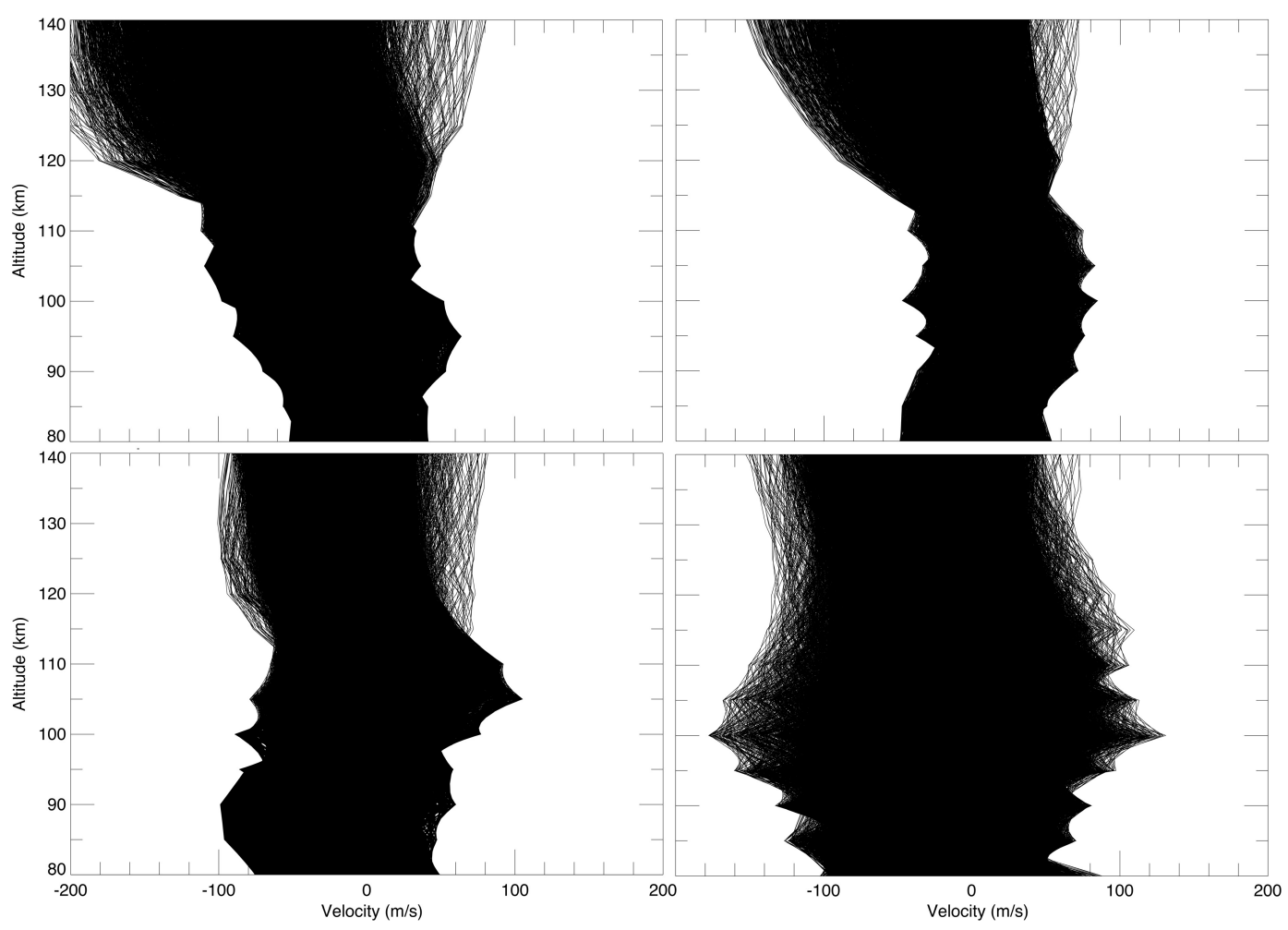

Fig. 6. Superposition of wind profiles for all TIME-GCM grid points between $52.5^{\circ} \mathrm{S}$ and $52.5^{\circ} \mathrm{N}$ for four different model runs. The top left panel shows the results with constant solar and geomagnetic forcing. The top right panel shows the results for variable solar and geomagnetic forcing. The lower left panel shows the curves when NCEP forcing is included at the 30-km lower boundary of the model. The lower right panel shows the profiles obtained when the model height resolution is doubled.

\section{Comparisons of individual nights}

As a final comparison, we show the model winds and the observations on two different nights. The agreement between individual profiles compared in this way is expected to be poor, but the comparisons will provide an indication of the extent to which the general features of the profiles are found in the model profiles.

The first comparison uses a sequence of six wind profiles measured with the chemical release technique on 1718 November 1965, from a launch site in Barbados. An extensive list of the data sources for the chemical release wind profiles is given by Larsen (2002). The sequence of wind profiles is plotted in the top panel of Fig. 8 and shows characteristics that are common in many of the wind profiles, namely tidal-like oscillations that propagate downward with time. The amplitude of the oscillations increases as the structure propagates through the $95-115-\mathrm{km}$ altitude range. The maximum winds are larger than predicted by tidal theory. The six corresponding HWM wind profiles are plotted in the lower panel of the figure. The model winds are much smaller than the observed winds and do not show the downward propagation of the structure. The vertical shears are too small as well. The lack of detailed agreement in this case is not surprising since the empirical model uses global fits to the available data that are likely to smooth the small-scale structure, but the comparison gives an indication of the difference between the observed and model wind profile structure that can be expected.

The final comparison uses a chemical release wind profile obtained from a launch at Eglin Air Force Base in Florida on 3 December 1962, as a reference. The Eglin example is primarily shown as an illustration of how the winds for an individual location change in the model simulations as the model forcing and height resolution change. General conclusions about the ability of the model to reproduce individual profiles would require more than one or two comparisons and would require simulations that use the conditions appropriate for the year of the measurement. The observed wind components are shown as the heavier solid curves in Fig. 9, with the zonal component in the upper panel and the meridional component in the lower panel. The wind profiles for the four TIME-GCM model runs described in the previous section are shown as the lighter lines in the figure. Specifically, the model wind profiles for the nearest grid point and day of the year were used, although the model run was made for 

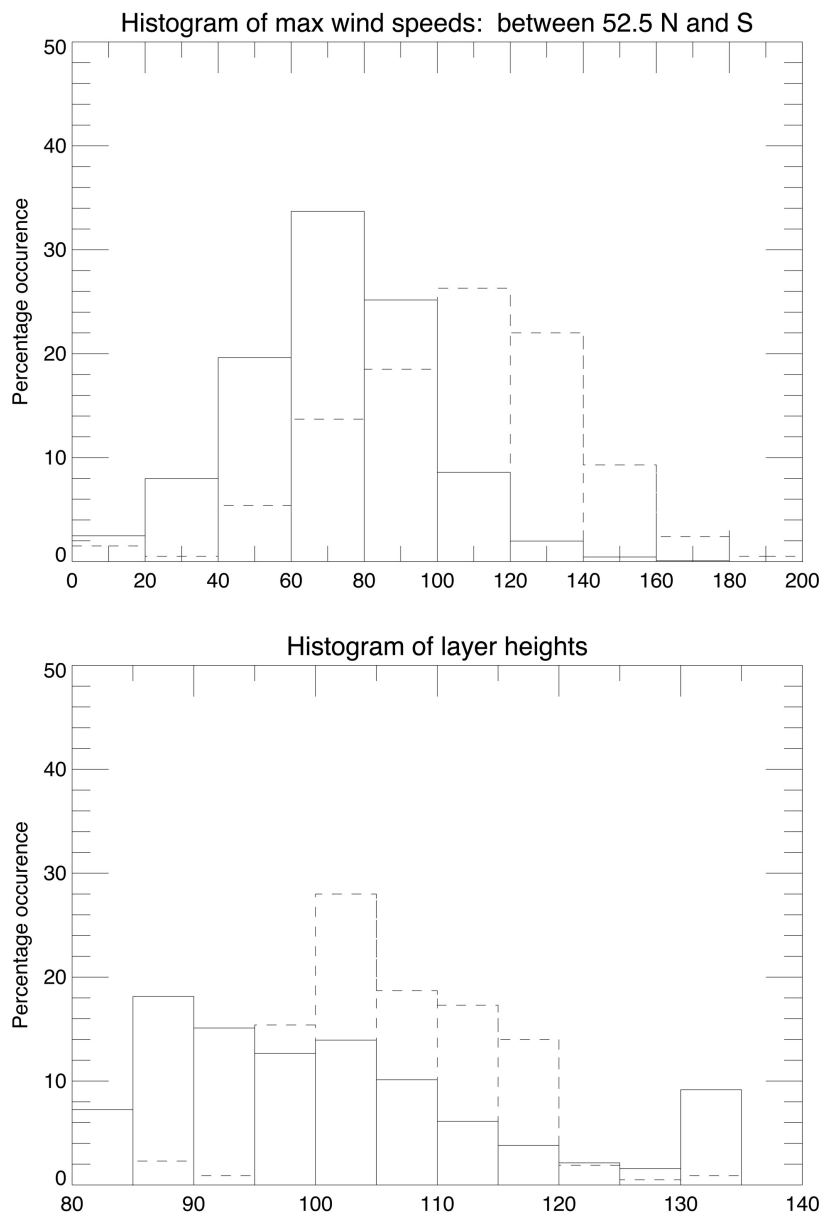

Fig. 7. Histograms of the observed winds (dashed curves) and model winds (solid curves) for the run with doubled height resolution. The top panel shows the distribution of maximum wind speeds. The lower panel shows the distribution of heights where the maximum winds occur.

parameters appropriate to 1993 rather than 1962, which was the year of the observation. The profiles of greatest interest in this case are the ones represented by the dash-dot line, which shows the results for the fourth run with doubled height resolution. Comparison of the model profiles for the four runs shows that the model is able to produce winds that are much more realistic, in terms of both the wind speeds and vertical shears, when the height resolution is increased. In particular, a feature similar to the observed eastward wind maximum near $105 \mathrm{~km}$ in the observations appears in that run, and the variation of the winds with height below the wind maximum is in good agreement with the observed zonal wind profile. The vertical variations of the meridional winds with height in the doubled-resolution model run are also in good qualitative agreement with the observations, although the maxima in the model meridional winds occur at a higher altitude than in the observations.
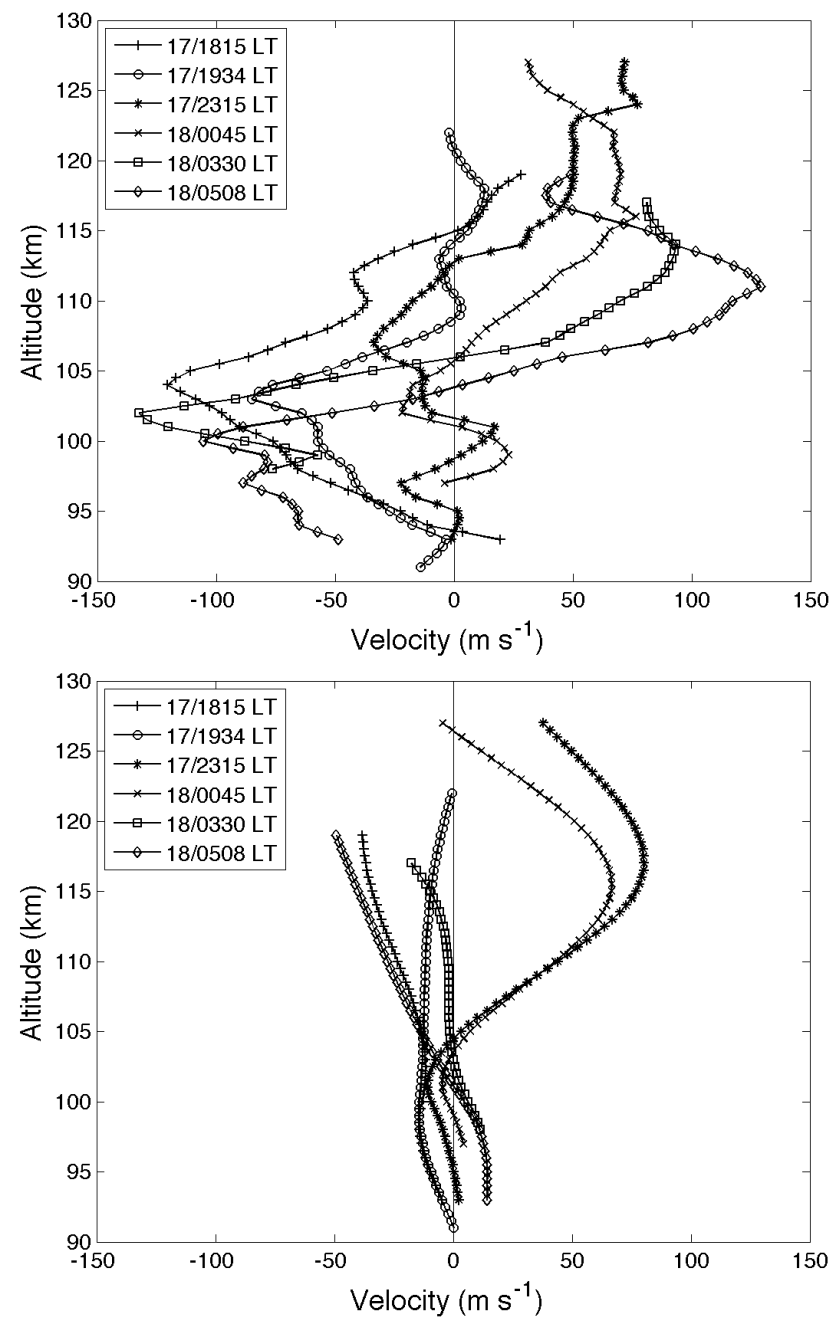

Fig. 8. Chemical release wind profiles obtained at Barbados during the night of 17-18 November 1965 are shown in the top panel. The lower panel shows the corresponding Horizontal Wind Model profiles.

\section{Conclusions}

The comparisons carried out here are by no means a detailed assessment of the validity of either the empirical or general circulation model. As discussed above in the description of the individual comparisons that are presented here, the GCM runs were carried out specifically for the year 1993 but were compared here with wind measurements made in different years or over a range of years. The GCM code has also been updated several times since the runs presented here and various versions of the model are currently available. There is also a new version of HWM that includes new data as input to the empirical model fits, including the rocket wind data set. The effect of such improvements in the models remains to be determined. 


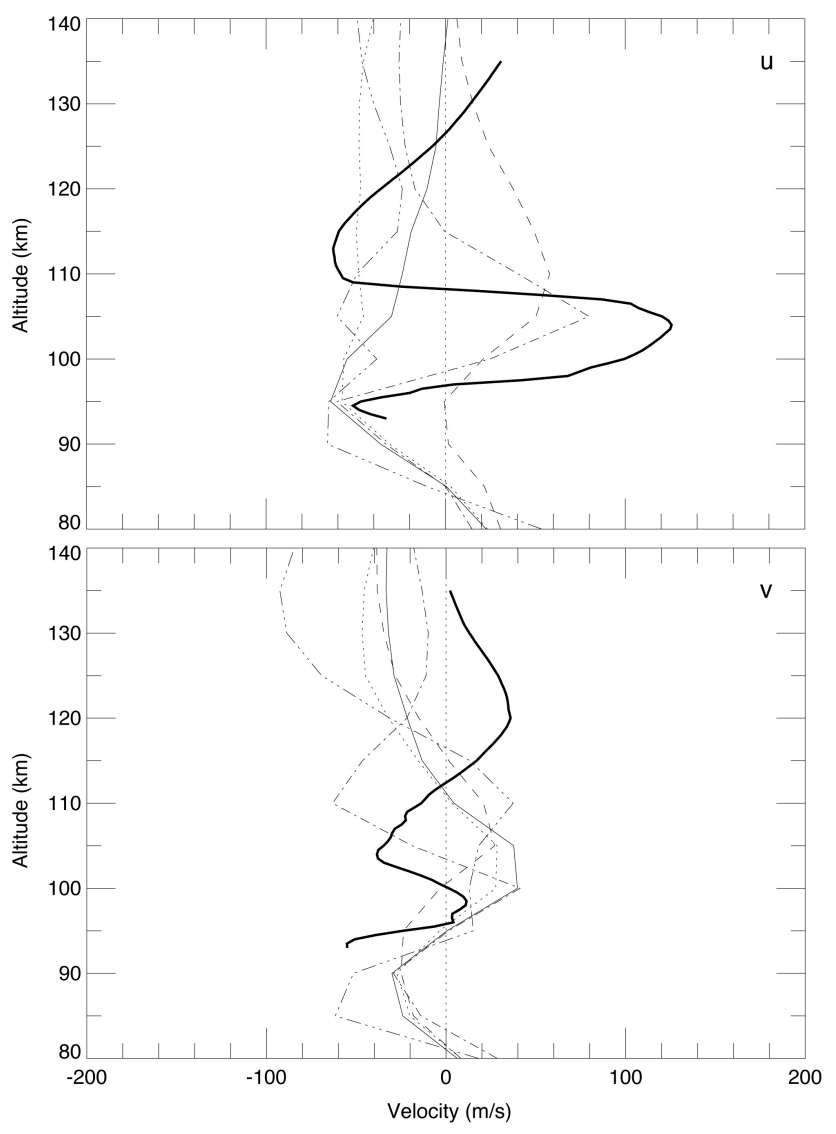

Fig. 9. Zonal (top panel) and meridional (lower panel) chemical release wind profiles obtained at Eglin Air Force Base in Florida at 22:30 UT on 3 December 1962 compared with the wind profiles for the nearest grid point and local time from the four TIME-GCM model runs. The wind profiles are shown for fixed forcing (solid line), varying solar and auroral forcing (dotted line), NCEP forcing (dashed line), and doubled height resolution (dot-dash line).

Our comparisons nonetheless show the extent to which the models can be expected to produce realistic wind maxima and vertical shears in the critical region in the lower Eregion. Our results suggest that the Horizontal Wind Model can produce mean winds that are in good agreement with the mean winds for the observational data set and wind variability that is within the one standard deviation limit in the observations. However, the shears and maximum winds produced by HWM significantly underestimate the observed values.

The most surprising result of the analysis presented here is that the TIME-GCM results improve significantly when the height resolution is doubled and lead to much better qualitative agreement between the model and the observations in the sense that the basic features of the enhanced lowerthermosphere winds are reproduced with realistic maximum winds and vertical wind variations. The mechanism responsible for the wind enhancement is still not known, but the fact that it appears in the model results indicates that it can be accounted for by the dynamics encompassed in the model. The large winds and shears therefore cannot be due to subgrid scale wave structure, i.e., wave structures with horizontal scales less than $\sim 1000 \mathrm{~km}$ in this case. The results therefore suggest that the mechanism is related either to dynamical processes that occur in the altitude range where the enhanced winds occur or to dynamical processes associated with larger-scale modes propagating upward from below.

Acknowledgements. The work at Clemson was partially supported by NSF grant ATM-0541593 and NASA grant NNX07AJ99G. Thanks to Ray Roble for performing the TIME-GCM simulations and providing the output.

Topical Editor K. Kauristie thanks S. Vadas and two other anonymous referees for their help in evaluating this paper.

\section{References}

Fesen, C. G., Hagan, M. E., Tepley, C. A., and Roble, R. G.: On the coupling between the lower and the upper thermosphere during the first Lower Thermosphere Coupling Study, J. Geophys. Res., 98, 1545-1558, 1993.

Hedin, A. E., Biondi, M. A., Burnside, R. G., Hernandez, G., Johnson, R. M., Killeen, T. L., Mazaudier, C., Meriwether, J. W., Salah, J. E., Sica, R. J., Smith, R. W., Spencer, N. W., Vickwar, V. B., and Virdi, T. S.: Revised global model of thermosphere winds using satellite and ground-based observations, J. Geophys. Res., 96, 7657-7688, 1991.

Larsen, M. F.: Winds and shears in the mesosphere and lower thermosphere: Results from four decades of chemical release wind measurements, J. Geophys. Res., 107(A8), 1215, doi:10.1029/2001JA000218, 2002.

Larsen, M. F., Liu, A. Z., Bishop, R. L., and Hecht, J. H.: TOMEX: A comparison of lidar and sounding rocket chemical tracer wind measurements, Geophys. Res. Lett., 30, 1375, doi:10.1029/2002GL015678, 2003.

Larsen, M. F., Hysell, D. L., Zhou, Q. H., Smith, S. M., Friedman, J., and Bishop, R. L.: Imaging coherent scatter radar, incoherent scatter radar, and optical observations of quasi-periodic structures associated with sporadic E layers, J. Geophys. Res., 112, A06321, doi:10.1029/2006JA012051, 2007.

Roble, R. G. and Ridley, E. C.: The Thermosphere-IonosphereMesosphere-Electrodynamic General Circulation Model (TIMEGCM): Equinox solar-cycle minimum simulations (30-500 km), Geophys. Res. Lett., 24, 417-420, 1994.

Vadas, S. L.: Horizontal and vertical propagation and dissipation of gravity waves in the thermosphere from lower atmospheric and thermospheric sources, J. Geophys. Res., 112, A06305, doi:10.1029/2006JA011845.

Zhou, X.: Analysis of wind speed and wind shear statistics and small scale structure using Doppler lidar data from New Mexico and Hawaii, Masters thesis, Clemson University, Clemson, South Carolina, 60 pp., 2005. 